\title{
RELATIVE NATURAL CLASSES AND RELATIVE INJECTIVITY
}

\author{
SEPTIMIU CRIVEI
}

Received 20 October 2004 and in revised form 24 January 2005

Let $\tau$ be a hereditary torsion theory on the category $R$-Mod of left $R$-modules over an associative unitary ring $R$. We introduce the notion of $\tau$-natural class as a class of modules closed under $\tau$-dense submodules, direct sums, and $\tau$-injective hulls. We study connections between certain conditions involving $\tau$-(quasi-)injectivity in the context of $\tau$ natural classes, generalizing results established by S. S. Page and Y. Q. Zhou (1994) for natural classes.

\section{Introduction and preliminaries}

The language of natural classes of modules appeared in the early 1990s, allowing to unify similar results that hold for some important classes of modules, such as the category $R$ Mod of left $R$-modules, any hereditary torsion-free class of modules, or any stable hereditary torsion class of modules. First, Dauns [2,3] introduced and studied natural classes for nonsingular modules under the name of saturated classes and, afterwards, Page and Zhou $[5,6]$ began the study of arbitrary natural classes.

In this paper, we are interested in relativizing the notion of a natural class to the torsion-theoretic framework and discussing connections between conditions on $\tau$ injectivity and $\tau$-quasi-injectivity in the context of $\tau$-natural classes, where $\tau$ is an arbitrary hereditary torsion theory on $R$-Mod.

Now we set the notation and terminology. The reference for general module theory is [8], whereas [1] and [4] will be mainly followed for torsion theories topics. Throughout, $R$ will be an associative ring with nonzero identity and all modules will be left unital $R$ modules. We will denote by $\tau$ a hereditary torsion theory on the category $R$-Mod of left $R$-modules.

If $\tau$ and $\sigma$ are two hereditary torsion theories such that every $\tau$-torsion module is $\sigma$-torsion, then it is said that $\sigma$ is a generalization of $\tau$ and it is denoted by $\tau \leq \sigma$. A submodule $B$ of a module $A$ is called $\tau$-dense in $A$ if $A / B$ is $\tau$-torsion. A torsion theory $\tau$ is called noetherian if for every ascending chain $I_{1} \subseteq I_{2} \subseteq \cdots$ of left ideals of $R$, the union of which is $\tau$-dense in $R$, there exists a positive integer $k$ such that $I_{k}$ is $\tau$-dense in 
$R$. A nonzero module $A$ is called $\tau$-cocritical if $A$ is $\tau$-torsion-free and each of its nonzero submodules is $\tau$-dense in $A$.

A module is said to be $\tau$-injective if it is injective with respect to every monomorphism having a $\tau$-torsion cokernel. For any module $A, E(A)$ and $E_{\tau}(A)$ denote the injective hull of $A$ and the $\tau$-injective hull of $A$, respectively. A module $A$ is said to be $\tau$-quasi-injective if whenever $B$ is a $\tau$-dense submodule of $A$, every homomorphism $B \rightarrow A$ extends to an endomorphism of $A$. A module is $\tau$-quasi-injective module if and only if it is a fully invariant submodule of its $\tau$-injective hull [7, Theorem 4.4]. A $\tau$-quasi-injective hull of a module $B$ is defined as a $\tau$-quasi-injective module $A$ such that $B$ is a $\tau$-dense essential submodule of $A$. Every module $A$ has a $\tau$-quasi-injective hull, unique up to an isomorphism [1, Propositions 5.1.8]. A module $A$ is called $\sum-\tau$-(quasi-)injective if every direct sum of copies of $A$ is $\tau$-(quasi-)injective.

A nonempty class $\mathscr{K}$ of modules is called a natural class if $\mathscr{K}$ is closed under isomorphic copies, submodules, direct sums, and injective hulls [5, page 2912]. Motivated by the torsion-theoretic context, a nonzero module $A$ is said to be $\mathscr{K}$-cocritical if $A \in \mathscr{K}$ and for every nonzero proper submodule $B$ of $A, A / B \notin \mathscr{K}[5$, page 2913].

\section{2. $\tau$-(quasi)-injectivity conditions for $\tau$-natural classes}

Throughout we will denote by $\mathscr{K}$ a nonempty class of modules closed under isomorphic copies. We will give the following definition.

Definition 2.1. The class $\mathscr{K}$ is called a $\tau$-natural class if $\mathscr{K}$ is closed under $\tau$-dense submodules, direct sums, and $\tau$-injective hulls.

Clearly, every natural class is a $\tau$-natural class.

Example 2.2. (i) The category $R$-Mod, any hereditary torsion-free class of modules and any stable hereditary torsion class of modules are natural classes, hence $\tau$-natural classes.

(ii) Let $\sigma$ be a hereditary torsion theory such that $\tau \leq \sigma$. Then the class of all $\sigma$-torsion modules is a $\tau$-natural class, that is, a natural class if and only if $\sigma$ is stable.

First we establish some necessary or sufficient conditions under which every direct sum of $\tau$-injective modules in $\mathscr{Y}$ is $\tau$-injective.

Theorem 2.3. Let $\mathscr{Y}$ be a $\tau$-natural class and suppose that every direct sum of $\tau$-injective modules in $\mathscr{K}$ is $\tau$-injective. Then every ascending chain $I_{1} \subseteq I_{2} \subseteq \cdots$ of $\tau$-dense left ideals of $R$ such that each $I_{j+1} / I_{j} \in \mathscr{K}$ terminates.

Proof. Suppose that $I_{1} \subset I_{2} \subset \cdots$ is a strictly ascending chain of $\tau$-dense left ideals of $R$ such that each $I_{j+1} / I_{j} \in \mathscr{K}$. By hypothesis, $E=\bigoplus_{j} E_{\tau}\left(I_{j+1} / I_{j}\right) \in \mathscr{K}$ is $\tau$-injective. Let $I=\bigcup_{j=1}^{\infty} I_{j}$, let $p_{j}: I_{j+1} \rightarrow I_{j+1} / I_{j}$ be the natural homomorphism, and let $\alpha_{j}: I_{j+1} / I_{j} \rightarrow$ $E_{\tau}\left(I_{j+1} / I_{j}\right)$ be the inclusion homomorphism for each $j$. Since $I_{j+1}$ is $\tau$-dense in $R$, the $\tau$-injectivity of $E_{\tau}\left(I_{j+1} / I_{j}\right)$ assures the existence of a homomorphism $\beta_{j}: R \rightarrow E_{\tau}\left(I_{j+1} / I_{j}\right)$ 
that extends $\alpha_{j} p_{j}$. Hence we have the following commutative diagram:

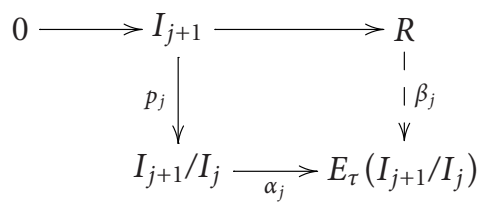

We may define $f: I \rightarrow E$ by $f(x)=\left(\beta_{j}(x)\right)_{j}$ for every $x \in I$. It is easy to check that $f$ is a well-defined homomorphism. Since $I$ is $\tau$-dense in $R$ and $E$ is $\tau$-injective, there exists a homomorphism $g: R \rightarrow E$ that extends $f$. Since $g(1) \subseteq \sum_{j=1}^{n} E_{\tau}\left(I_{j+1} / I_{j}\right)$ for some positive integer $n$, we have

$$
f(I)=g(I) \subseteq \sum_{j=1}^{n} E_{\tau}\left(\frac{I_{j+1}}{I_{j}}\right) .
$$

It follows that $\beta_{j}(x)=0$ for every $x \in I$ and every $j>n$. If $x \in I_{n+1}$, then $0=\beta_{n+1}(x)=$ $x+I_{n}$. Hence $I_{n+1}=I_{n}$, a contradiction.

Remark 2.4. Note that in the proof of Theorem 2.3, each $I_{j+1} / I_{j}$ is $\tau$-torsion. Hence we have used only the fact that every direct sum of $\tau$-torsion $\tau$-injective modules in $\mathscr{K}$ is $\tau$-injective.

Proposition 2.5. Let $\mathscr{K}$ be a $\tau$-natural class and suppose that every ascending chain $I_{1} \subseteq$ $I_{2} \subseteq \cdots$ of left ideals of $R$ whose union is $\tau$-dense in $R$ such that each $I_{j+1} / I_{j} \in \mathscr{K}$ terminates. Then every direct sum of $\tau$-injective modules in $\mathscr{K}$ is $\tau$-injective.

Proof. It is sufficient to prove that every countable direct sum of $\tau$-injective modules in $\mathscr{K}$ is $\tau$-injective (see [4, page 384]). Let $A=\bigoplus_{i=1}^{\infty} A_{i}$ be a countable direct sum of $\tau$-injective modules in $\mathcal{K}$. Also let $I$ be a $\tau$-dense left ideal of $R$ and let $f: I \rightarrow A$ be a homomorphism. For each $n$, denote

$$
I_{n}=\left\{x \in I \mid f(x) \in \bigoplus_{i=1}^{n} A_{i}\right\} .
$$

Clearly $I_{1} \subseteq I_{2} \subseteq \cdots$ and $\bigcup_{j=1}^{\infty} I_{j}=I$. We may consider the monomorphism

$$
\alpha_{n}: \frac{I_{n+1}}{I_{n}} \longrightarrow \frac{\left(\bigoplus_{i=1}^{n+1} A_{i}\right)}{\left(\bigoplus_{i=1}^{n} A_{i}\right)}
$$

defined by $\alpha_{n}\left(x+I_{n}\right)=f(x)+\left(\bigoplus_{i=1}^{n} A_{i}\right)$ for every $x \in I_{n+1}$. Since the codomain of $\alpha_{n}$ is isomorphic to $A_{n+1} \in \mathscr{K}$, we have $I_{n+1} / I_{n} \in \mathscr{K}$. By hypothesis, there is a positive integer $k$ such that $I_{k+j}=I_{k}$ for each $j$. Then $f(I) \subseteq \bigoplus_{i=1}^{k} A_{i}$. Since $\bigoplus_{i=1}^{k} A_{i}$ is $\tau$-injective, there exists a homomorphism $g: R \rightarrow \bigoplus_{i=1}^{k} A_{i} \subseteq A$ that extends $f$. Thus $A$ is $\tau$-injective. 
Corollary 2.6. Let $\tau$ be noetherian and $\mathscr{Y}$ a $\tau$-natural class. Then the following statements are equivalent.

(i) Every direct sum of $\tau$-injective modules in $\mathscr{K}$ is $\tau$-injective.

(ii) Every ascending chain $I_{1} \subseteq I_{2} \subseteq \cdots$ of $\tau$-dense left ideals of $R$ such that each $I_{j+1} / I_{j} \in \mathscr{K}$ terminates.

Proof. (i) $\Rightarrow$ (ii) by Theorem 2.3 .

(ii) $\Rightarrow$ (i). Let $I_{1} \subseteq I_{2} \subseteq \cdots$ be an ascending chain of left ideals of $R$ whose union is $\tau$ dense in $R$ such that each $I_{j+1} / I_{j} \in \mathcal{K}$. Since $\tau$ is noetherian, there exists a positive integer $k$ such that $I_{k}$ is $\tau$-dense in $R$. Then $I_{n}$ is $\tau$-dense in $R$ for every $n \geq k$. By hypothesis, the chain $I_{k} \subseteq I_{k+1} \subseteq \cdots$ terminates, hence the chain $I_{1} \subseteq I_{2} \subseteq \cdots I_{k} \subseteq I_{k+1} \subseteq \cdots$ terminates. Now use Proposition 2.5.

Following [5], denote by $H_{\mathscr{K}}(R)$ the set of left ideals $I$ of $R$ such that $R / I \in \mathscr{K}$.

THEOREM 2.7. Let $\mathscr{K}$ be a $\tau$-natural class and suppose that every ascending chain $I_{1} \subseteq I_{2} \subseteq$ $\cdots$ of $\tau$-dense left ideals of $R$ such that each $I_{j+1} / I_{j} \in \mathscr{K}$ terminates. Then $H_{\mathscr{K}}(R)$ has ACC on $\tau$-dense left ideals.

Proof. Let $I_{1} \subseteq I_{2} \subseteq \cdots$ be an ascending chain of $\tau$-dense left ideals in $H_{\mathscr{K}}(R)$. Then each $R / I_{j} \in \mathscr{K}$. Since $\mathscr{K}$ is closed under $\tau$-dense submodules, each $I_{j+1} / I_{j} \in \mathscr{K}$. By hypothesis, the above chain terminates.

In what follows, we will establish connections between some conditions involving $\tau$ quasi-injective modules in the context of $\tau$-natural classes.

We need the following lemma, that generalizes a classical result for quasi-injective modules.

Lemma 2.8. A module $A$ is $\tau$-injective if and only if $A \oplus E_{\tau}(A)$ is $\tau$-quasi-injective.

Proof. The direct implication is obvious. Suppose now that $A \oplus E_{\tau}(A)$ is $\tau$-quasi-injective. Consider the exact sequence $0 \rightarrow A \stackrel{i}{\rightarrow} E_{\tau}(A) \stackrel{p}{\rightarrow} E_{\tau}(A) / A \rightarrow 0$, where $i$ is the inclusion homomorphism and $p$ is the natural homomorphism. Denote $j=1_{A} \oplus i$ and let $\alpha_{1}: A \rightarrow$ $A \oplus E_{\tau}(A)$ and $\alpha_{2}: E_{\tau}(A) \rightarrow A \oplus E_{\tau}(A)$ be the inclusions into $A$ and $E_{\tau}(A)$, respectively, let $\beta: A \rightarrow A \oplus A$ be the inclusion into the second summand and let $\sigma: A \oplus A \rightarrow A \oplus E_{\tau}(A)$ be defined by $\sigma\left(a_{1}, a_{2}\right)=\left(a_{2}, a_{1}\right)$ for every $\left(a_{1}, a_{2}\right) \in A \oplus A$. Now consider the following commutative diagram:

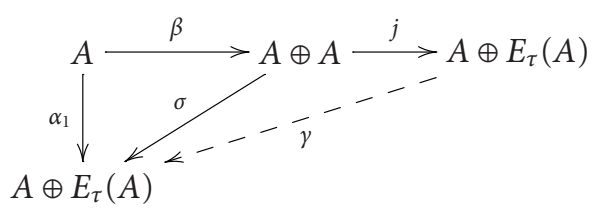

Since $A \oplus A$ is $\tau$-dense in $E_{\tau}(A \oplus A)=E_{\tau}(A) \oplus E_{\tau}(A)$, it follows that $A \oplus A$ is $\tau$-dense in $A \oplus E_{\tau}(A)$. But $A \oplus E_{\tau}(A)$ is $\tau$-quasi-injective, hence there exists a homomorphism $\gamma: A \oplus E_{\tau}(A) \rightarrow A \oplus E_{\tau}(A)$ such that $\gamma j=\sigma$. Now let $\pi: A \oplus E_{\tau}(A) \rightarrow A$ be the projection 
and take $\delta=\pi \gamma \alpha_{2}$. Then we have $\delta i=\pi \gamma \alpha_{2} i=\pi \gamma j \beta=\pi \sigma \beta=\pi \alpha_{1}=1_{A}$, hence $A$ is a direct summand of $E_{\tau}(A)$. But $A$ is essential in $E_{\tau}(A)$, hence we must have $A=E_{\tau}(A)$, showing that $A$ is $\tau$-injective.

Theorem 2.9. Let $\mathscr{Y}$ be a $\tau$-natural class. The following statements are equivalent.

(i) Every direct sum of $\tau$-quasi-injective modules in $\mathscr{K}$ is $\tau$-quasi-injective.

(ii) Every direct sum of $\tau$-injective modules in $\mathscr{K}$ is $\tau$-injective and every $\tau$-quasi-injective module in $\mathscr{Y}$ is $\tau$-injective.

Proof. (i) $\Rightarrow$ (ii). Let $A \in \mathscr{K}$ be a $\tau$-quasi-injective module. Since $\mathscr{K}$ is a $\tau$-natural class, $E_{\tau}(A) \in \mathscr{K}$. By hypothesis, $A \oplus E_{\tau}(A)$ is $\tau$-quasi-injective. Now by Lemma $2.8, A$ is $\tau$ injective. Therefore every $\tau$-quasi-injective module in $\mathscr{K}$ is $\tau$-injective.

Now let $A=\bigoplus_{i \in I} A_{i}$, where each $A_{i}$ is a $\tau$-injective module in $\mathscr{K}$. Hence each $A_{i}$ is a $\tau$-quasi-injective module in $\mathscr{K}$. By hypothesis, $A$ is a $\tau$-quasi-injective module in $\mathscr{K}$. By the first part, $A$ is a $\tau$-injective module in $\mathscr{K}$. Therefore every direct sum of $\tau$-injective modules in $\mathscr{K}$ is $\tau$-injective.

(ii) $\Rightarrow$ (i). Let $A=\bigoplus_{i \in I} A_{i}$, where each $A_{i}$ is a $\tau$-quasi-injective module in $\mathscr{K}$. By hypothesis, each $A_{i}$ is a $\tau$-injective module in $\mathscr{K}$ and $A$ is a $\tau$-injective module in $\mathscr{K}$. Hence $A$ is a $\tau$-quasi-injective module in $\mathscr{K}$.

Now consider the following condition.

(C) For every ascending chain $I_{1} \subseteq I_{2} \subseteq \cdots$ of left ideals of $H_{\mathscr{K}}(R), \bigcup_{j=1}^{\infty} I_{j} \in H_{\mathscr{K}}(R)$ [5].

For the reader's convenience, we recall some preliminary lemmas. We will consider the same definition of a $\mathscr{Y}$-cocritical module for a $\tau$-natural class $\mathscr{Y}$ as in the case of a natural class $\mathscr{Y}$.

Lemma 2.10 (see [5, Lemma 4]). Let $\mathscr{Y}$ be a natural class and let $A$ be a $\mathscr{Y}$-cocritical module. Then $A$ is uniform and any nonzero homomorphism from a submodule of $A$ to a module of $\mathscr{K}$ is a monomorphism. In particular, the class of $\mathscr{Y}$-cocritical modules is closed under nonzero submodules.

Lemma 2.11 (see [5, Lemma 6]). Let $\mathscr{Y}$ be a natural class. If condition (C) holds, then every cyclic module in $\mathscr{K}$ has a $\mathscr{K}$-cocritical homomorphic image.

Lemma 2.12 (see [5, Lemma 7]). Let $A$ be a module and let $a_{1}, \ldots, a_{n} \in A$. If all homomorphic images of $R a_{1}, \ldots, R a_{n}$ which are submodules of $E(A)$ have finite uniform dimension, then $E\left(R a_{1}\right)+\cdots+E\left(R a_{n}\right)$ has finite uniform dimension.

Now we are able to prove the following result, but for a natural class $\mathscr{K}$.

Theorem 2.13. Let $\mathscr{Y}$ be a natural class and suppose that condition $(C)$ holds and every $\tau$-quasi-injective module in $\mathscr{Y}$ is $\tau$-injective. Then $H_{\mathscr{K}}(R)$ has ACC on $\tau$-dense left ideals.

Proof. Suppose that $I_{1} \subset I_{2} \subset \cdots$ is a strictly ascending chain of $\tau$-dense left ideals of $H_{\mathscr{K}}(R)$. Then $I_{j+1} / I_{j} \in \mathscr{K}$ for each $j$. By Lemma 2.11, there exist $U_{j}$ and $V_{j+1}$ such that $I_{j} \subseteq$

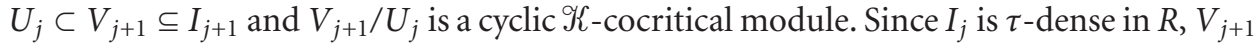
is $\tau$-dense in $R$, so that $V_{j+1} / U_{j}$ is $\tau$-dense in $R / U_{j}$. Now let $\alpha_{j}: V_{j+1} / U_{j} \rightarrow E_{\tau}\left(V_{j+1} / U_{j}\right)$ be the inclusion homomorphism for each $j$. By the $\tau$-injectivity of $E_{\tau}\left(V_{j+1} / U_{j}\right)$, there exists a homomorphism $\beta_{j}: R / U_{j} \rightarrow E_{\tau}\left(V_{j+1} / U_{j}\right)$ that extends $\alpha_{j}$. Denote $I=\bigcup_{j=1}^{\infty} I_{j}$ and 
$A=\bigoplus_{j} E_{\tau}\left(V_{j+1} / U_{j}\right)$. Since $E_{\tau}\left(V_{j+1} / U_{j}\right) \in \mathscr{K}$, we have $A \in \mathscr{K}$. We may define $f: I \rightarrow A$ by $f(x)=\left(\beta_{j}\left(x+U_{j}\right)\right)_{j}$ for every $x \in I$. It is easy to check that $f$ is a well-defined homomorphism. Let

$$
Q=\sum\left\{h(A) \mid h \in \operatorname{End}_{R}\left(E_{\tau}(A)\right)\right\}
$$

be the $\tau$-quasi-injective hull of $A$ (see [1, Proposition 5.1.7]). We have $E_{\tau}(A) \in \mathscr{K}$, hence $Q \in \mathscr{K}$. Then $Q$ is $\tau$-injective. Since $I$ is $\tau$-dense in $R$, there exists a homomorphism $g: R \rightarrow Q$ such that the following diagram, where the unspecified homomorphisms are inclusions, is commutative:

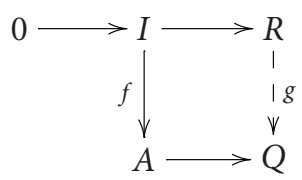

Then we have $g(1) \in N=\sum_{k=1}^{t} \sum_{j=1}^{s} h_{k}\left(E_{\tau}\left(V_{j+1} / U_{j}\right)\right)$ for some positive integers $t$ and $s$, whence it follows that $f(I) \subseteq N$. By Lemma $2.10, h_{k}\left(V_{j+1} / U_{j}\right) \cong V_{j+1} / U_{j}$ is a cyclic $\mathscr{K}$ cocritical module. Moreover, $E_{\tau}\left(h_{k}\left(V_{j+1} / U_{j}\right)\right)=h_{k}\left(E_{\tau}\left(V_{j+1} / U_{j}\right)\right)$. By Lemma 2.12 and again by Lemma 2.10, $N$ has a finite uniform dimension.

On the other hand, $E_{\tau}\left(f\left(V_{2}\right)\right)=E_{\tau}\left(V_{2} / U_{1}\right)$ and $f\left(V_{2}\right) \subseteq f\left(V_{3}\right) \subseteq E_{\tau}\left(V_{2} / U_{1}\right) \oplus V_{3} / U_{2}$. Since $f\left(V_{3}\right) \nsubseteq E_{\tau}\left(f\left(V_{2}\right)\right)$ and all $V_{j+1} / U_{j}$ are uniform, it follows that $E_{\tau}\left(f\left(V_{3}\right)\right)=$ $E_{\tau}\left(V_{2} / U_{1}\right) \oplus E_{\tau}\left(V_{3} / U_{2}\right)$. Similarly, for each positive integer $n$, we have

$$
E_{\tau}\left(f\left(V_{n}\right)\right)=E_{\tau}\left(\frac{V_{2}}{U_{1}}\right) \oplus E_{\tau}\left(\frac{V_{3}}{U_{2}}\right) \oplus \cdots \oplus E_{\tau}\left(\frac{V_{n+1}}{U_{n}}\right) .
$$

But this means that $E(f(I))$ and, consequently, $f(I)$ have infinite uniform dimension, a contradiction.

Proposition 2.14. Let $\sigma$ be a hereditary torsion theory such that $\tau \leq \sigma$ and let $\mathscr{Y}$ be the class of all $\sigma$-torsion modules. Suppose also that every $\tau$-quasi-injective module in $\mathscr{Y}$ is $\tau$-injective. Then $H_{\%}(R)$ has ACC on $\tau$-dense left ideals.

Proof. Clearly, $\mathscr{K}$ is a $\tau$-natural class. Note also that the set of all $\sigma$-dense left ideals of $R$ is exactly $H_{\mathscr{K}}(R)$, hence condition (C) holds for $\mathscr{K}$. Let $A$ be a $\mathscr{K}$-cocritical module. If there exists a nonzero submodule $B$ of $A$, then $A / B$ is $\sigma$-torsion, that is, $A / B \in \mathscr{K}$, a contradiction. Hence $A$ is simple and thus uniform. Therefore every $\mathscr{Y}$-cocritical module is simple.

We mention that Lemma 2.11 holds for this particular $\tau$-natural class $\mathscr{K}$, the proofs being identical. Note also that since every $\tau$-torsion module is $\sigma$-torsion, the set of $\tau$ dense left ideals of $R$ is contained in $H_{\mathscr{K}}(R)$. Now the result follows by the same arguments as in the proof of Theorem 2.13.

The following result on $\tau$-quasi-injective modules will be useful.

Lemma 2.15. Let $A$ be a $\tau$-quasi-injective module. If $\left(E_{\tau}(A)\right)^{(I)}$ is $\tau$-injective, then $A^{(I)}$ is $\tau$-quasi-injective for every set $I$. 
Proof. The proof is immediate, using the fact that if $C$ is a fully invariant submodule of a module $B$, then $C^{(I)}$ is a fully invariant submodule of $B^{(I)}$ for every set $I$.

Theorem 2.16. Let $\mathscr{Y}$ be a $\tau$-natural class and suppose that every $\tau$-quasi-injective module in $\mathscr{Y}$ is $\sum$ - $\tau$-quasi-injective. Then $H_{\mathscr{K}}(R)$ has ACC on $\tau$-dense left ideals.

Proof. Let $I_{1} \subseteq I_{2} \subseteq \cdots$ be an ascending chain of $\tau$-dense left ideals of $R$ such that each $I_{j} \in \mathscr{H}_{\mathscr{K}}(R)$. Denote $E_{j}=E_{\tau}\left(R / I_{j}\right)$ and $A=\bigoplus_{j=1}^{\infty} E_{j}$. Clearly each $E_{j} \in \mathcal{K}$, hence $A \in \mathscr{K}$. Let $p_{j}: A \rightarrow E_{j}$ be the projection and consider the following diagram, where the unspecified homomorphisms are inclusions:

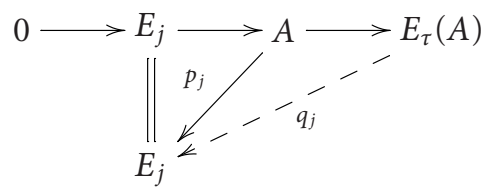

Since $A$ is $\tau$-dense in $E_{\tau}(A)$ and $E_{j}$ is $\tau$-injective, there exists a homomorphism $q_{j}$ : $E_{\tau}(A) \rightarrow E_{j}$ that extends $p_{j}$. Then $E_{j}$ is a direct summand of $E_{\tau}(A)$, hence $E_{\tau}(A)=E_{j} \oplus C_{j}$ for some submodule $C_{j}$ of $E_{\tau}(A)$. We have

$$
\left(E_{\tau}(A)\right)^{(\mathbb{N})} \cong \bigoplus_{j=1}^{\infty}\left(E_{j} \oplus C_{j}\right)=\left(\bigoplus_{j=1}^{\infty} E_{j}\right) \oplus\left(\bigoplus_{j=1}^{\infty} C_{j}\right)=A \oplus\left(\bigoplus_{j=1}^{\infty} C_{j}\right) .
$$

By hypothesis, $\left(E_{\tau}(A)\right)^{(\mathbb{N})}$ is $\tau$-quasi-injective, hence $A$ is $\tau$-quasi-injective by Lemma 2.15 . Denote $I=\bigcup_{j=1}^{\infty} I_{j}$. For each $j$, define a homomorphism $f_{j}: I / I_{1} \rightarrow E_{j}$ by $f_{j}\left(x+I_{1}\right)=$ $x+I_{j}$ for every $x \in I$. Then we may define a homomorphism $f: I / I_{1} \rightarrow A$ by $f\left(x+I_{1}\right)=$ $\left(f_{j}(x)\right)_{j}$ for every $x \in I$. It is easy to check that $f$ is well defined. Consider the following diagram, where the unspecified homomorphisms are inclusions:

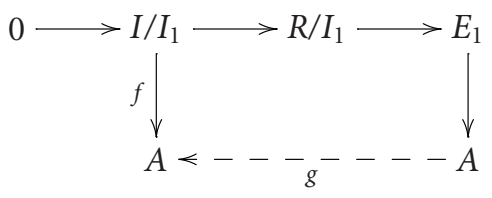

Note that $I$ is $\tau$-dense in $R$, hence $I / I_{1}$ is $\tau$-dense in $R / I_{1}$. Clearly, $R / I_{1}$ is $\tau$-dense in $E_{1}$. Further, $A / E_{1} \cong \bigoplus_{j=2}^{\infty} E_{j}$ is $\tau$-torsion because each $E_{j}=E_{\tau}\left(R / I_{j}\right)$ is $\tau$-torsion. Hence $E_{1}$ is $\tau$-dense in $A$. It follows that $I / I_{1}$ is $\tau$-dense in $A$. Now since $A$ is $\tau$-quasi-injective, there exists a homomorphism $g: A \rightarrow A$ that extends $f$. It follows that $f\left(I / I_{1}\right) \subseteq g\left(R / I_{1}\right) \subseteq A$. Since $a=g\left(1+I_{1}\right) \in A$, we have $f\left(I / I_{1}\right) \subseteq R a \subseteq \bigoplus_{j=1}^{n} E_{j}$ for some positive integer $n$. Then $I_{n+1}=I_{n+2}=\cdots=I$.

Theorem 2.17. Let $\mathscr{Y}$ be a $\tau$-natural class and suppose that every $\tau$-injective module in $\mathscr{K}$ is $\sum$ - $\tau$-injective. Then every $\tau$-quasi-injective module in $\mathscr{K}$ is $\sum$ - $\tau$-quasi-injective.

Proof. Let $A$ be a $\tau$-quasi-injective module in $\mathscr{K}$ and let $I$ be a set. By hypothesis, $\left(E_{\tau}(A)\right)^{(I)}$ is $\tau$-injective. Then by Lemma $2.15, A^{(I)}$ is $\tau$-quasi-injective. Hence $A$ is $\sum$ - $\tau$-quasiinjective. 
Theorem 2.18. Let $\mathcal{K}$ be a natural class. The following statements are equivalent.

(i) Every $\tau$-injective module in $\mathscr{K}$ is injective.

(ii) Every $\tau$-quasi-injective module in $\mathscr{Y}$ is quasi-injective.

Proof. (i) $\Rightarrow$ (ii). Let $A$ be a $\tau$-quasi-injective module in $\mathscr{Y}$. Then $A$ is a fully invariant submodule of $E_{\tau}(A)$. But $E_{\tau}(A)=E(A)$. Hence $A$ is a fully invariant submodule of $E(A)$, that is, $A$ is quasi-injective.

(ii) $\Rightarrow$ (i). Let $A$ be a $\tau$-injective module in $\mathscr{Y}$. Then $A$ is a $\tau$-quasi-injective module in $\mathscr{K}$, hence $A$ is quasi-injective by hypothesis. Clearly, $A \oplus E(A) \in \mathscr{K}$. Moreover, $A \oplus E(A)$ is $\tau$-injective, hence $\tau$-quasi-injective. By hypothesis, $A \oplus E(A)$ is quasi-injective. Now by Lemma 2.8 applied for the improper torsion theory (i.e., the torsion theory whose torsion class consists of all modules), it follows that $A$ is injective.

Remark 2.19. If $\mathscr{K}$ is a natural class and $\tau$ is the improper torsion theory, Theorems 2.3, 2.7, 2.9, 2.13, 2.16 yield results of Page and Zhou $[5,6]$.

\section{Acknowledgment}

The author was partially supported by the Grant 344/2004 CNCSIS, Romania.

\section{References}

[1] P. E. Bland, Topics in Torsion Theory, Mathematical Research, vol. 103, Wiley-VCH Verlag Berlin $\mathrm{GmbH}$, Berlin, 1998.

[2] J. Dauns, Classes of modules, Forum Math. 3 (1991), no. 4, 327-338.

[3] Direct sums and subdirect products, Methods in Module Theory (Colorado Springs, CO, 1991), Lecture Notes in Pure and Appl. Math., vol. 140, Dekker, New York, 1993, pp. 39-65.

[4] J. S. Golan, Torsion Theories, Pitman Monographs and Surveys in Pure and Applied Mathematics, vol. 29, Longman Scientific \& Technical, Harlow, 1986.

[5] S. S. Page and Y. Q. Zhou, Direct sums of quasi-injective modules, injective covers, and natural classes, Comm. Algebra 22 (1994), no. 8, 2911-2923.

[6] On direct sums of injective modules and chain conditions, Canad. J. Math. 46 (1994), no. 3, 634-647.

[7] C. L. Walker and E. A. Walker, Quotient categories and rings of quotients, Rocky Mountain J. Math. 2 (1972), no. 4, 513-555.

[8] R. Wisbauer, Foundations of Module and Ring Theory, Algebra, Logic and Applications, vol. 3, Gordon and Breach Science Publishers, Pennsylvania, 1991.

Septimiu Crivei: Department of Mathematics, Faculty of Mathematics and Computer Science, Babeş-Bolyai University, 1 Mihail Kogălniceanu Street, 400084 Cluj-Napoca, Romania

E-mail address: crivei@math.ubbcluj.ro 


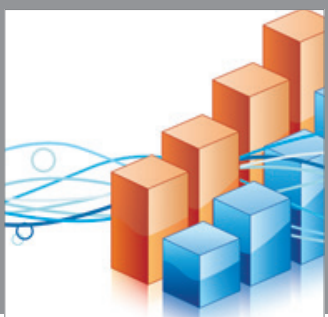

Advances in

Operations Research

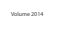

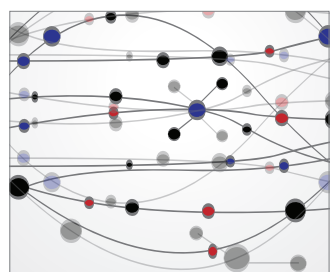

\section{The Scientific} World Journal
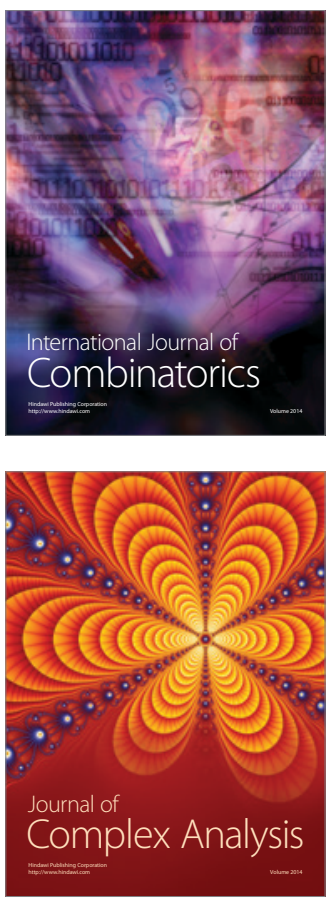

International Journal of

Mathematics and

Mathematical

Sciences
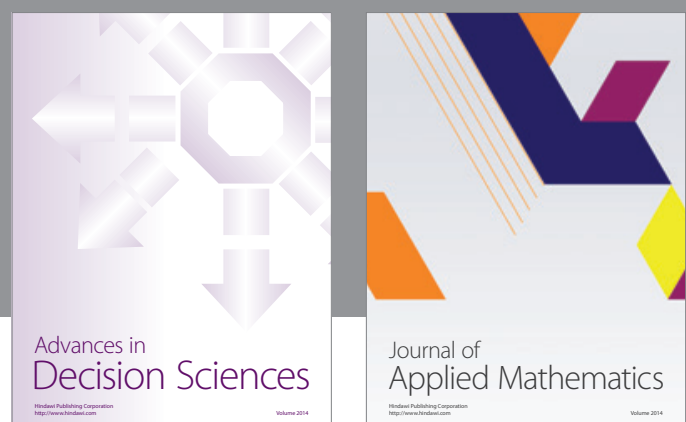

Journal of

Applied Mathematics
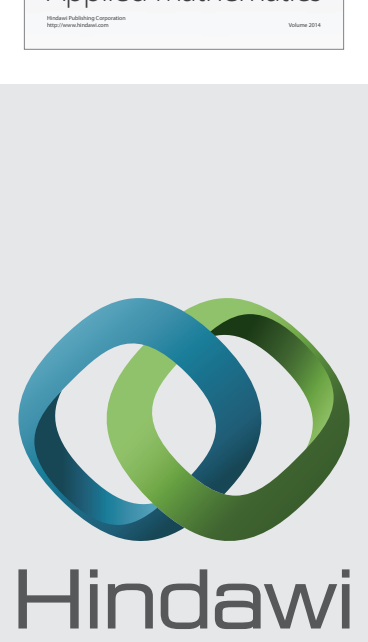

Submit your manuscripts at http://www.hindawi.com
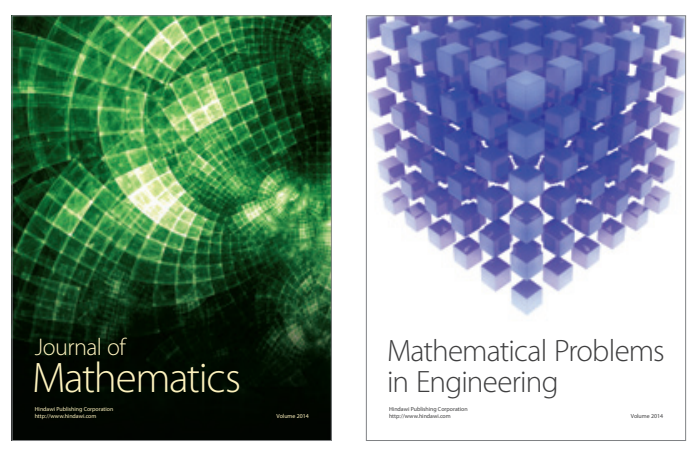

Mathematical Problems in Engineering
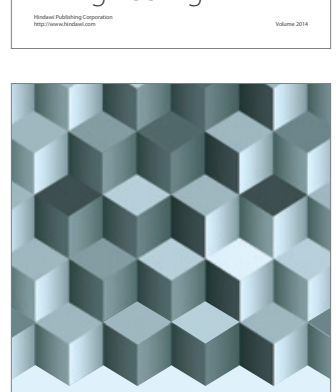

Journal of

Function Spaces
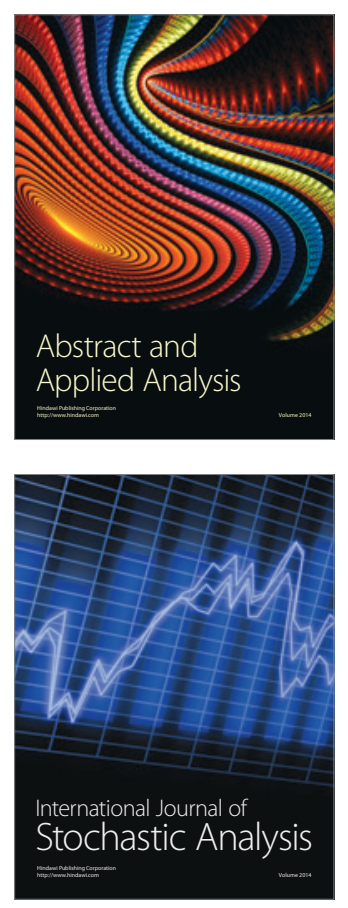

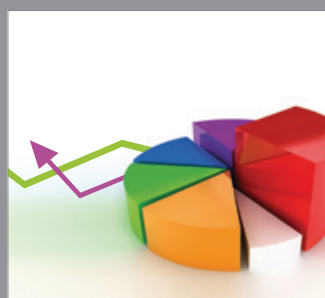

ournal of

Probability and Statistics

Promensencen
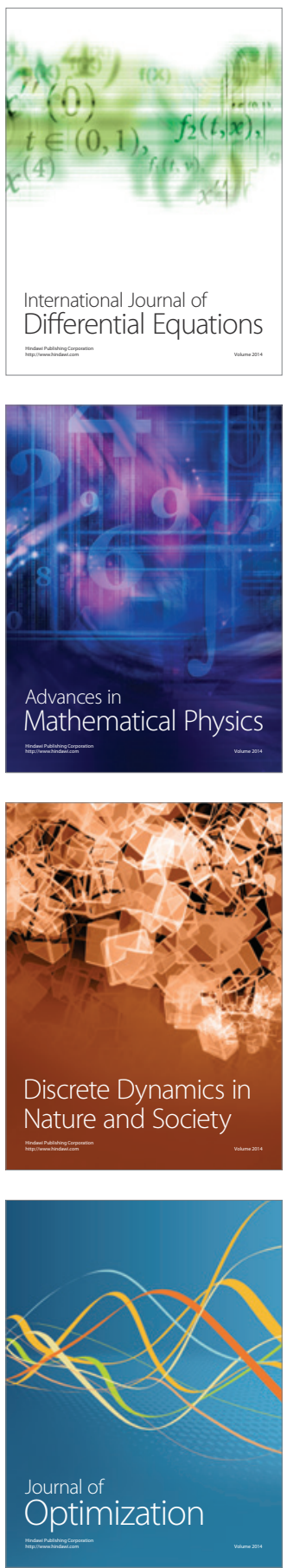